\title{
COMPARAÇÃO DE MÉTODOS DE PROGNOSE DA ESTRUTURA DIAMÉTRICA DE UMA FLORESTA ESTACIONAL SEMIDECIDUAL SECUNDÁRIA ${ }^{1}$
}

\author{
Suely Lundreeng Austregésilo², Rinaldo Luiz Caraciolo Ferreira ${ }^{3}$, José Antônio Aleixo da Silva³, Agostinho Lopes de \\ Souza $^{4}$, Isabelle Maria Jacqueline Meunier ${ }^{5}$ e Eufrázio de Souza Santos ${ }^{5}$
}

\begin{abstract}
RESUMO - Objetivou-se comparar prognoses da distribuição diamétrica de uma floresta estacional semidecidual secundária obtidas pelos métodos da razão de movimentação, de Wahlenberg e da matriz de transição. Foram utilizados dados obtidos em dez anos de monitoramento do ensaio de produção sustentável em floresta secundária, implantado em 1986 pela companhia Vale do Rio Doce, em Rio Vermelho e Serra Azul de Minas, Minas Gerais. Foram considerados apenas os dados referentes à área sem intervenção, e as árvores foram agrupadas por classe de diâmetro de $5 \mathrm{~cm}$. As análises da taxa de crescimento foram realizadas a partir dos períodos de monitoramento de 1986 a 1996, com intervalos de dois em dois anos. As prognoses da estrutura diamétrica para 1994 e 1996 foram realizadas por meio dos métodos da razão de movimentação, de Wahlenberg e da matriz de transição e comparadas com a estrutura real. A consistência da prognose obtida por cada método foi realizada por meio do teste de Kolmogorov-Smirnov $(\mathrm{P}=0,05)$. Quanto ao número médio de árvores, as prognoses obtidas pelos três métodos foram similares a estrutura real, tanto para 1994 como para 1996. No entanto, quanto à distribuição diamétrica, os métodos não foram eficientes quando comparados com a estrutura real. Pode-se concluir que os métodos da razão de movimentação, de Wahlenberg e da matriz de transição foram eficientes na prognose do número médio de árvores/ha da floresta estudada, porém não o foram na prognose da distribuição diamétrica da mesma floresta.
\end{abstract}

Palavras-chave: Matriz de transição, método de Wahlenberg e razão de movimentação.

\section{COMPARISON OF METHODS OF DIAMETER DISTRIBUTION PROGNOSIS OF A SEASONAL SECONDARY SEMIDECIDUAL FOREST}

\begin{abstract}
This research aimed to compare prognoses of the diameter distribution of a seasonal secondary semidecidual forest using the movement ratio, Wahlenberg and the transition methods. The data used was obtained during a 10-year survey of sustainable production in a secondary forest implanted in 1986 by the Vale do Rio Doce Company in Vermelho and Serra Azul de Minas, Minas Gerais, Brazil. Only the data regarding an area without intervention was used and the trees were grouped by class of $5 \mathrm{~cm}$ diameter. Growth ratio analyses were carried out from 1986 to 1996 every two years. The diameter structure prognoses for 1994 and 1996 were made by using the movement ratio, Wahlenberg and Matrix transition methods by comparing with the actual structure. To test the consistency of the prognosis obtained by each method, the Kolmogorov-Smirnov test $(P=0.05)$ was used. For the mean number of trees, the prognoses obtained by the three methods were similar to the actual structure, in 1994 and 1996. However, for diameter distribution, the methods were not efficient when compared with the actual structure. It was concluded that the movement ratio, Wahlenberg and matrix transition methods were efficient in prognosing the mean number of trees/ha; the methods movement ratio, however they were not efficient in prognosing diameter distribution.
\end{abstract}

Key words: Transition matrix, Wahlenberg's method, movement ratio method.

Recebido para publicação em 9.1.2003 e aceito para publicação em 30.4.2004.

Bióloga, M.S. em Biometria. ${ }^{3}$ Professor Adjunto da Universidade Federal Rural de Pernambuco - UFRPE, Bolsista CNPq, <rinaldof@ufrpe>, <jaaleixo@uol.com.br>. ${ }^{4}$ Professor Titular da Universidade Federal de Viçosa - UFV, Bolsista CNPq, <alsouza@ufv.br>. ${ }^{5}$ Professor(a) Adjunto da UFRPE, <meunier@hotlink,com.br>, <eufrazio@ufrpe.br> 


\section{INTRODUÇÃO}

As florestas devem ser estudadas para propiciar o conhecimento e a manutenção da biodiversidade, assim como para que se viabilize a exploração de seus produtos, bens e ou serviços provenientes de forma planejada e racional, para garantir o fluxo contínuo desses recursos.

A carência de um planejamento sistemático pode levar à definição de objetivos a partir de critérios subjetivos e à reduzida compatibilidade entre ações e objetivos. O planejamento é essencial no manejo florestal, em razão do longo tempo envolvido e também das dificuldades de promover mudanças bruscas na condução de uma floresta, sem alterar a sustentabilidade (Schneider \& Finger, 2000).

Os modelos de crescimento e produção são técnicas usadas para prognosticar a dinâmica de um povoamento, e independentemente da complexidade estrutural que possam apresentar todos os modelos de crescimento e produção têm um propósito em comum, que é o de apresentar estimativas das características do povoamento de um ponto específico no tempo (Avery \& Burkhart, 1994).

Para Vanclay (1995), apesar dos avanços conseguidos, a modelagem do crescimento e produção florestal é mais uma arte do que uma ciência, apesar dos vários modelos sugeridos em literatura. Muitos modelos são excessivamente empíricos, pois há maior preocupação na validação do modelo do que na compreensão das teorias biológicas.

Segundo Vanclay (1994), se for levada em consideração a hierarquia dos modelos, estes se distinguem em: modelos de gerenciamento florestal, de povoamento, de classe de diâmetro e de árvores individuais.

Os modelos de distribuição diamétrica são os mais comuns, e se baseiam em funções probabilísticas de distribuição, permitindo descrever as alterações na estrutura do povoamento (número de árvores por classe de diâmetro), nas relações hipsométricas e nas taxas de mortalidade, podendo todas estas características ser analisadas, simultaneamente, ao longo do tempo. Nesse tipo de modelo destacam-se:

a) As tabelas de povoamento ou produção, nas quais se encontram o método da razão de movimentação e o método de Wahlenberg.

b) Os modelos estocásticos de crescimento em diâmetro, em que se tem a matriz de transição.

R. Árvore, Viçosa-MG, v.28, n.2, p.227-232, 2004
Esses modelos são intermediários entre os modelos de povoamento total e os de árvores individuais, e podem fornecer informações suficientes para o manejo de florestas naturais inequiiâneas, sem a complexidade inerente aos modelos de árvores individuais. Os modelos de classe de tamanho incluem projeção de tabelas de povoamento, matrizes de transição e modelos de corte.

Portanto, o presente trabalho teve por objetivo avaliar a acuracidade da predição da distribuição diamétrica de uma floresta estacional semidecidual secundária por meio de métodos de predição. Especificamente, procurou-se:

1) Aplicar os métodos da razão de movimentação, de Wahlenberg e da matriz de transição na predição da estrutura diamétrica.

2) Comparar a predição da estrutura diamétrica pelos métodos utilizados com a estrutura real.

\section{MATERIALEMÉTODOS}

Para este estudo foram utilizados dados obtidos em dez anos de monitoramento do ensaio de produção sustentável em floresta secundária, implantado em 1986 pela companhia Vale do Rio Doce. Maiores detalhes podem ser encontrados em Ferreira et al. (1999).

Neste trabalho foram utilizados apenas os dados referentes ao tratamento sem intervenção, e as árvores foram agrupadas por classe de diâmetro de $5 \mathrm{~cm}$. Foram obtidas nove classes, que abrangeram desde o centro da classe 7,5, composta de árvores de $5 \mathrm{a} 10 \mathrm{~cm}$, até as árvores no centro da classe de $47,5 \mathrm{~cm}$, composta de árvores de 45 a $50 \mathrm{~cm}$.

As análises da taxa de crescimento foram realizadas a partir dos períodos de monitoramento de 1986 a 1996, com intervalos de dois em dois anos.

\subsection{Modelos de Crescimento e Produção Empregados}

Os períodos considerados foram de 1986 a 1990 e 1988 a 1992. O ingresso e a mortalidade não foram modelados para todos os métodos de prognose, e sim utilizados diretamente da base de dados.

\subsubsection{Método da Razão de Movimentação}

Para os cálculos do método da razão de movimentação foram analisados os dados referentes ao diâmetro 
das árvores, nos períodos de 1986 a 1990 e 1988 a 1992. $\mathrm{O}$ incremento periódico individual das árvores foi obtido pela expressão:

$$
I P D_{i}=D A P_{2 i}-D A P_{1 i}
$$

em que $I P D_{i}=$ incremento periódico em diâmetro da iésima árvore no período de avaliação; $D A P_{1 i}=D A P$ da iésima árvore no início do período de avaliação; e $D A P_{2 i}=$ $D A P$ da i-ésima árvore no final do período de avaliação (i $=1,2, \ldots, n)$.

O incremento periódico médio por classe diamétrica foi calculado de acordo com a expressão:

$$
\overline{I P D}_{j}=\frac{\sum_{i, j=1}^{n, N}\left(D A P_{2 i j}-D A P_{1 i j}\right)}{N_{j}}
$$

em que $\overline{I P D}_{j}=$ incremento periódico médio em diâmetro na j-ésima classe diamétrica, $\operatorname{com} j=1,2, \ldots, \mathrm{N} ; D A P_{1 i j}=$ $D A P$ da i-ésima árvore, na j-ésima classe de diâmetro, no início do período de avaliação; $D A P_{2 i j}=D A P$ da i-ésima árvore, na j-ésima classe de diâmetro, no final do período de avaliação; $\mathrm{N}_{\mathrm{j}}=$ número de árvores na j-ésima classe de diâmetro; e $D A P_{1 i j}$ e $D A P_{2 i j} \rightarrow D A P$ da i-ésima árvore na j-ésima classe de diâmetro no início e no final do período de avaliação, respectivamente.

Como ingresso foram consideradas todas as árvores que no segundo inventário passaram a compor a amostra, ou seja, todas as árvores que na segunda medição atingiram o $D A P$ mínimo de $5,0 \mathrm{~cm}$.

A mortalidade foi obtida a partir do segundo inventário, através da contagem de árvores em suas respectivas classes.

Na razão de movimentação assume-se que as árvores estão distribuídas uniformemente no interior das classes, onde cada árvore cresce a uma taxa média. A distribuição das árvores no interior das classes é desconhecida, mesmo assim assume-se que ela é uniforme. A partir daí a proporção de árvores que passam de uma classe para outra pode ser assumida como razão de movimento, tendo-se:

$$
R M=\left(\frac{\overline{I P D_{j}}}{C}\right) 100
$$

em que $R M=$ razão de movimento; $\overline{I P D}_{j}=$ incremento periódico médio em diâmetro da j-ésima classe de diâmetro; e $C=$ amplitude de classe de diâmetro $(5 \mathrm{~cm})$.

Na interpretação da razão de movimentação (RM), os primeiros dois dígitos da direita indicam a porcentagem de árvores que avançam uma classe de diâmetro em relação ao terceiro dígito à direita. Por exemplo, a RM $=24,14 \%$ para a classe $7,5 \mathrm{~cm}$, no período de 1986 a 1990 , permite indicar que 24,14\% das árvores existentes em 1986 moveram-se para a classe $12,5 \mathrm{~cm}$ e que $75,86 \%$ das árvores permaneceram na classe em 1990 .

\subsubsection{Método de Wahlenberg}

Na aplicação deste método obteve-se a variação do incremento diamétrico individual das árvores, no interior de cada classe. Neste caso, a porcentagem de movimento foi calculada ao aplicar o incremento individual no período sobre o diâmetro das árvores (Husch et al., 1993).

Foram contabilizados os avanços das árvores nas classes diamétricas por meio da identificação das árvores estacionárias (aquelas em que o incremento periódico não foi suficiente para mudança de classe), as que avançaram apenas uma classe e também as árvores que avançaram duas classes, no respectivo período analisado.

Com os cálculos das porcentagens de movimento para cada classe de diâmetro, a evolução da estrutura da floresta foi realizada do mesmo modo que a razão de movimentação, descrita anteriormente.

As informações sobre a mortalidade e o recrutamento foram obtidas de forma idêntica ao método da razão de movimentação.

\subsubsection{Matriz de Transição}

A estimação das probabilidades de transição foi realizada por simples proporção, uma vez que por meio da série de dados identificou-se o número de árvores por classe de diâmetro que, entre dois inventários, permaneceram na mesma classe, mudaram de classe, foram perdidas por mortalidade e ingressaram.

Portanto, as probabilidades, para cada intervalo de medição, foram obtidas ao dividir o número de árvores, respectivamente, que mudaram de classe ou permaneceram na mesma classe pelo número de árvores naquela classe no início do período de crescimento. Logo, o cálculo das probabilidades de transição foi realizado por meio da fórmula:

R. Árvore, Viçosa-MG, v.28, n.2, p.227-232, 2004 


$$
P_{i j}=\left(\frac{n_{i j}}{n_{i}}\right)
$$

em que $n_{i j}=$ número de indivíduos na classe $\mathrm{j}$, no tempo $t+1$, uma vez que estavam na classe $\mathrm{i}$, no tempo $t$, para $i=$ $1, \ldots, k$ e $j=1, \ldots, k ; n_{j}=$ número total de indivíduos na classe $\mathrm{i}$, no tempo t.

Assim, a matriz de transição $(G)$ da cadeia de Markov é quadrada, k x k, com elementos $P_{i j}$ :

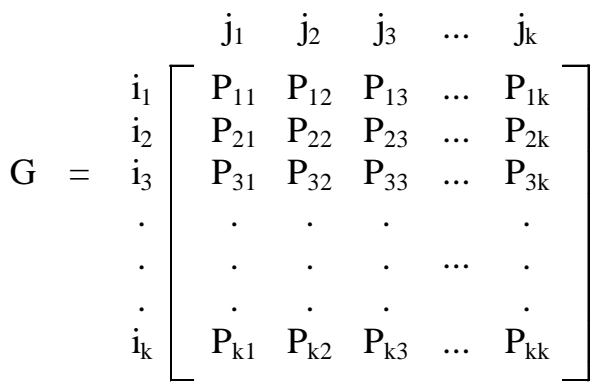

em que G é não-negativa e a soma $\mathrm{p}_{\mathrm{i} 1}+\mathrm{p}_{\mathrm{i} 2}+\ldots+\mathrm{p}_{\mathrm{ij}}=1$.

A diagonal principal da matriz $\mathrm{G}$ representa a proporção de árvores que permanecem na mesma classe de tamanho $\left(a_{i}\right)$; a subdiagonal representa a proporção das árvores que se movem para a próxima classe de tamanho $\left(b_{i}\right)$; abaixo da subdiagonal estão as proporções das árvores que avançaram mais de uma classe $\left(c_{i}\right)$, na unidade de tempo; e o resto da matriz é preenchido com zeros.

A projeção da estrutura da floresta foi realizada de acordo com Buongiorno \& Michie (1980), como se segue:

$$
Y_{t+? t}=G^{n} . Y_{i t}+I_{i t}
$$

em que $Y_{t+\Delta t}=$ número de árvores no tempo $t+\Delta t ; G=$ matriz de probabilidade de transição por classe diamétrica; $Y_{i t}=$ vetor número de árvores por classe de diâmetro no tempo $t$, com dimensão $1 \mathrm{x} k ; l_{i t}=$ vetor número de árvores ingresso no tempo $t$, com dimensão $1 \mathrm{x} k$; e $\mathrm{n}$ $=n$ período de prognose.

\subsection{Avaliação da Projeção}

Inicialmente foi realizada uma análise de variância, considerando como tratamentos a estrutura real (testemunha) e as prognosticadas conforme o método utilizado. As classes diamétricas foram consideradas como os blocos.

A consistência da prognose obtida por cada método, avaliando se a distribuição diamétrica prognosticada é similar à distribuição real, foi realizada por meio do teste de Kolmogorov-Smirnov, conforme sugerido por Sokal \& Rohlf (1995), a 0,05 do teste bilateral.

\section{RESULTADOS E DISCUSSÃO}

\subsection{Prognose da Estrutura da Floresta pelo Método da Razão de Movimentação}

No Quadro 1 está um resumo das prognoses obtidas pelos métodos da razão de movimentação, de Wahlenberg e da matriz de transição e real para 1994 e 1996, com base nos períodos de 1986 a 1992 e 1988 a 1992, respectivamente.

Nas prognoses da distribuição diamétrica da floresta para 1994 e 1996, pelo método da razão de movimentação, observa-se que o número de árvores na menor classe de diâmetro foi decrescendo ao longo das projeções. Este padrão também foi observado por Silva (1989) e Pulz (1998), que encontraram subestimativa do número de árvores na menor classe de diâmetro, entre o povoamento real e o projetado. Para as outras classes de diâmetro, observou-se a tendência de superestimativa do número de árvores. As maiores diferenças observadas entre a estrutura real e a prognose para 1994, possivelmente, se devem ao fato de que a floresta está em pleno crescimento, o que indica que a modelagem da estrutura da floresta só será realmente eficiente em todas as classes diamétricas se a modelagem do ingresso e da mortalidade forem conseguidas. Por outro lado, observa-se que a prognose da estrutura da floresta para 1996 é bem próxima em termos de número total de árvores/ha e que as discrepâncias dentro das classes são menores, o que pode estar associado ao maior equilíbrio entre as taxas de mortalidade e ingresso.

Nas prognoses da estrutura da floresta para $1994 \mathrm{e}$ 1996 pelo método de Wahlenberg observa-se que o número de árvores na menor classe de diâmetro foi superestimado ao longo das projeções, sendo, portanto, de comportamento oposto ao verificado pelo método da razão de movimentação (Quadro 1). Também nesse método, as maiores diferenças observadas entre a estrutura real e a prognose para 1994 podem estar associadas ao fato de que a floresta estava em pleno crescimento e que 
Quadro 1 - Resumo das prognoses da distribuição diamétrica por meio dos métodos da razão de movimentação, de Wahlenberg e da matriz de transição e real para 1994 e 1996, com base nos períodos de 1986 a 1992 e 1988 a 1992 , respectivamente

Table 1 - Summary of the prognoses of diameter distribution by applying the methods of movement ratio, Wahlenberg and transition and actual matrix for 1994 and 1996, based on $1986-1992$ and 1988-1992, periods, respectively

\begin{tabular}{|c|c|c|c|c|c|c|c|c|}
\hline \multirow{4}{*}{$\begin{array}{c}\text { Classe } \\
\text { Diamétrica } \\
(\mathrm{cm})\end{array}$} & \multicolumn{8}{|c|}{ Distribuição Diamétrica (Número de Árvores/ha) } \\
\hline & \multicolumn{4}{|c|}{1994} & \multicolumn{4}{|c|}{1996} \\
\hline & \multicolumn{3}{|c|}{ Método } & \multirow[b]{2}{*}{ Real } & \multicolumn{3}{|c|}{ Método } & \multirow[b]{2}{*}{ Real } \\
\hline & $\begin{array}{c}\text { Razão de } \\
\text { Movimentação }\end{array}$ & Wahlenberg & $\begin{array}{l}\text { Matriz de } \\
\text { Transição }\end{array}$ & & $\begin{array}{c}\text { Razão de } \\
\text { Movimentação }\end{array}$ & Wahlenberg & $\begin{array}{l}\text { Matriz de } \\
\text { Transição }\end{array}$ & \\
\hline 7,5 & $1.564,30$ & $1.756,84$ & $1.374,66$ & 1640,00 & $1.477,75$ & $1.650,93$ & $1.570,78$ & 1598,50 \\
\hline 12,5 & 694,37 & 591,94 & 639,00 & 512,50 & 581,03 & 542,96 & 518,44 & 528,00 \\
\hline 17,5 & 217,41 & 183,90 & 224,72 & 162,00 & 215,88 & 205,09 & 205,00 & 160,50 \\
\hline 22,5 & 53,42 & 42,97 & 51,81 & 38,50 & 51,52 & 44,54 & 44,54 & 41,50 \\
\hline 27,5 & 18,50 & 17,61 & 22,17 & 14,50 & 20,95 & 19,48 & 19,48 & 16,00 \\
\hline 32,5 & 7,73 & 7,17 & 8,36 & 6,50 & 7,79 & 6,33 & 6,32 & 8,00 \\
\hline 37,5 & 3,18 & 2,56 & 3,01 & 5,00 & 5,66 & 5,72 & 5,71 & 2,50 \\
\hline 42,5 & 1,49 & 1,67 & 2,18 & 0,50 & 1,29 & 0,80 & 0,80 & 2,50 \\
\hline 47,5 & 0,13 & & 0,00 & 0,50 & 0,16 & 0,50 & 0,00 & 0,50 \\
\hline Total & $2.560,50$ & $2.604,66$ & $2.235,91$ & 2380,00 & $2.362,00$ & $2.476,35$ & $2.371,07$ & $2.358,00$ \\
\hline
\end{tabular}

a modelagem da estrutura da floresta só será realmente eficiente em todas as classes diamétricas se a modelagem do ingresso e da mortalidade forem conseguidas. Observa-se também que a projeção da estrutura da floresta para 1996 é bem próxima em termos de número total de árvores/ha e que as discrepâncias dentro das classes são menores, o que pode estar associado ao maior equilíbrio entre mortalidade e ingresso.

Nas prognoses para 1994 e 1996 da estrutura da floresta pelo método da matriz de transição (Quadro 1) pode-se observar que a projeção do número total de árvores/ha, tanto para 1994 como para 1996, está bem próximo da estrutura real, sendo $6,05 \%$ menor que o real para 1994 e superior em 0,55\% para 1996. Vale salientar que as matrizes de transição para os períodos 86-90 e 88-92 apresentaram estados de absorção nas classes maiores, o que indica que o processo muda consideravelmente a cada passo, sendo impossível fazer previsões a longo prazo.

\subsection{Avaliação das Prognoses da Distribuição Diamétrica}

Por meio da análise de variância verificou-se a igualdade $(\mathrm{P}>0,05)$, em número médio de árvores, entre as estruturas prognosticadas pelos métodos e a real, tanto para 1994 como para 1996, o que significa que os métodos foram eficientes quanto à estimativa dessa variável.

Nas Figuras 1 e 2 pode-se observar que as prognoses têm a distribuição típica de floresta natural, que é do tipo J-invertido. No entanto, por meio do teste de Kolmogorov-Smirnov rejeita-se a hipótese de que a distribuição diamétrica prognosticada é similar à distribuição real $(\mathrm{P}<0,05)$, tanto para 1994 como para 1996, indicando

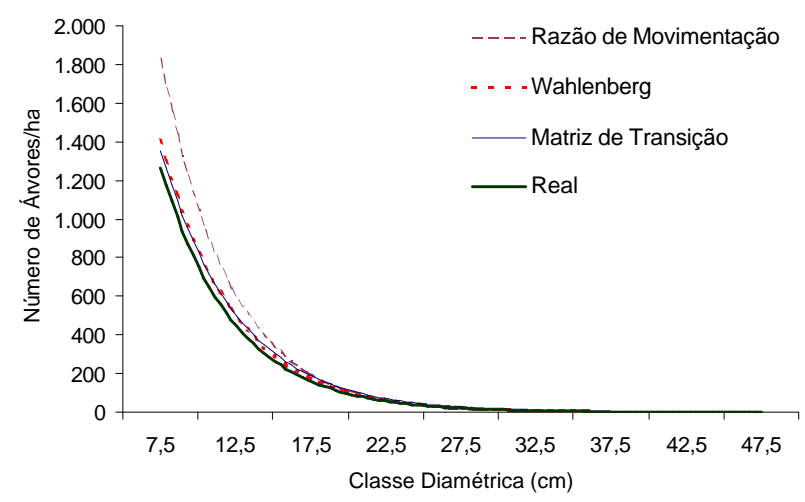

Figura 1 - Estrutura diamétrica real e prognosticada para 1994 conforme método aplicado.

Figure 1 - Actual and predicted diameter distribution for 1994, according to the applied method.

R. Árvore, Viçosa-MG, v.28, n.2, p.227-232, 2004 


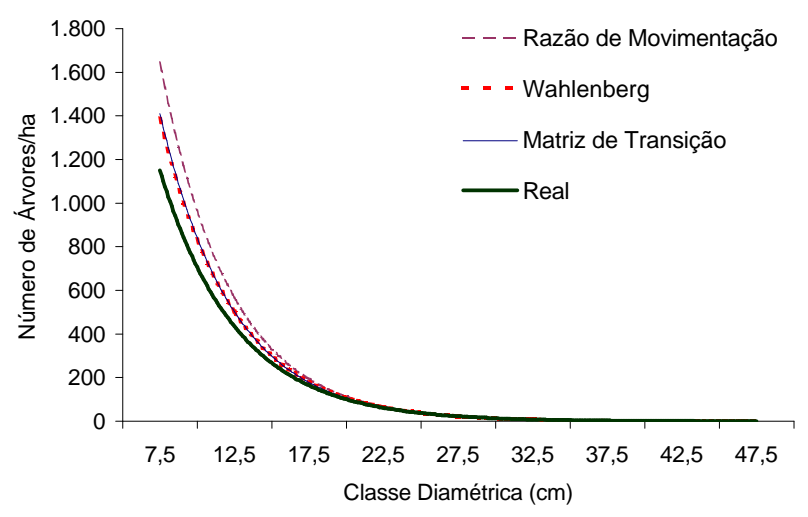

Figura 2 - Estrutura diamétrica real e prognosticada para 1996 conforme método aplicado.

Figure 2 - Actual and predicted diameter distribution for 1996, according to the applied method.

que os métodos aplicados não foram eficientes na prognose da distribuição diamétrica da floresta estudada. Vale ressaltar a similaridade das distribuições obtidas pelos métodos de Wahlenberg e da matriz de transição.

Deve-se salientar que neste estudo o ingresso não foi modelado e que os métodos poderão ser melhorados caso isso ocorra, aumentando a precisão das projeções a curto prazo e melhorando as projeções intermediárias e a longo prazo, para matrizes de probabilidades de transição estacionária.

\section{CONCLUSÕES}

- Os métodos da razão de movimentação, de Wahlenberg e da matriz de transição foram eficientes na prognose do número médio de árvores/ha da floresta estudada, porém não o foram na prognose da distribuição diamétrica da floresta estudada.

\section{REFERÊNCIAS BIBLIOGRÁFICAS}

AVERY, T. E.; BURKHART, H. E. Forest management. 4.ed. New York: McGraw-Hill, 1994, 432 p.

BUONGIORNO, J.; MICHIE, B. R. A matrix model of uneven-aged forest management. Forest Science, v. 26, p. $609-625,1980$.

HUSCH, B.; MILLER, C. I.; BEERS, T. W. Forest mensuration. Malabar: Krieger, 1993. 402 p.

FERREIRA, R. L. C.; SOUZA, A. L.; SILVA, G. F. Dinâmica da estrutura de uma floresta secundária de transição. III - Estrutura horizontal. Revista Árvore, v. 23 , n. 2, p. 157-168, 1999.

PULZ, F. A. Estudo da dinâmica e a modelagem de uma floresta semidecidua montana na região de Lavras-MG. 1998. 156 f. Dissertação (Mestrado em Engenharia Florestal) - Universidade Federal de Lavras, Lavras, 1998.

SCHNEIDER, P. R.; FINGER, C. A. G. Manejo sustentado de florestas inequiâneas heterogêneas. Santa Maria: UFSM, 2000. 99 p.

SILVA, J. N. M. The behavior of the tropical rain forest of the Brasilian Amazon after logging. 1989. $302 \mathrm{f}$. Thesis (Ph.D.) - Oxford University, Oxford, 1989.

SOKAL, R. R.; ROHLF, F. J. Biometry. 3.ed. New York: W.H. Freeman and Company, 1995. 887 p.

VANCLAY, J. K. A growth model for North Queensland rainforests. Forest Ecology and Management, v. 27, p. 245-271, 1994.

VANCLAY, J. K. Growth models for tropical forests: a synthesis of models and methods. Forest Science, v. 41, n. 1, p. 7-42, 1995. 